Unfallchirurg 2011 · 114:558

DOI 10.1007/s00113-010-1865-x

Online publiziert: 29. Juni 2011

(c) Springer-Verlag 2011

\author{
M. Schädel-Höpfner · J. Windolf \\ Klinik für Unfall- und Handchirurgie, Universitätsklinikum Düsseldorf
}

\title{
Gelenkverletzungen an der Hand
}

herstellung konnte mit den zur Verfügung stehenden traditionellen Operationstechniken wie Bohrdrahtosteosynthesen und Fixateur externe vielfach nicht erreicht werden.

Moderne Osteosyntheseverfahren erlauben eine präzisere Reposition und verlässlichere Stabilisierung der Fragmente, allerdings unter Inkaufnahme einer höheren Invasivität. Insbesondere anatomisch geformte, winkelstabile palmare Platten haben zu dieser Entwicklung beigetragen und prägen inzwischen die Behandlung distaler Radiusfrakturen, auch wenn ihre Überlegenheit gegenüber anderen Techniken bislang nicht nach evidenzbasierten Kriterien erwiesen wurde. Auch die Technik der winkelstabilen palmaren Plattenosteosynthese bedarf einer ständigen Überprüfung und Optimierung, z. B. durch die intraoperative Anwendung dreidimensionaler Abbildungssysteme.

Frakturen des distalen Radius können mit Luxationen des Karpus kombiniert sein. Derartige radiokarpale Luxationsfrakturen führen zu besonders schweren knöchernen und ligamentären Schädigungen des Handgelenks und der Handwurzel. Auch die Luxationsfrakturen zwischen der distalen Karpalreihe und den Metakarpalia stellen kritische Verletzungen dar, aus denen spezielle Anforderungen für die Untersuchung und Behandlung resultieren. Im Falle einer unzureichenden oder verspäteten Diagnostik bzw. Therapie drohen permanente, schmerzhafte Funktionseinbußen. Selbst bei einer idealen chirurgischen Versorgung ist eine Restitutio ad integrum nicht kalkulierbar. Obwohl grundsätzliche Empfehlungen für diese Verletzungen exisitieren, ist stets ein individuelles Therapiekonzept zu erstellen, dessen Umsetzung ein hohes Maß an handchirurgisch-traumatologischer Erfahrung erfordert. Darüber hinaus ist die realistische Einschätzung der Prognose für Patienten und Behandler wichtig. Deshalb halten wir die Darstellung der Besonderheiten sowohl der radiokarpalen als auch der karpometakarpalen Luxationsfrakturen für besonders relevant.

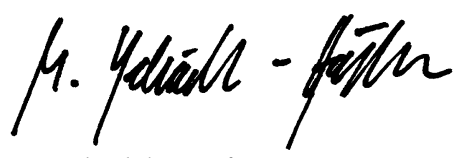

M. Schädel-Höpfner
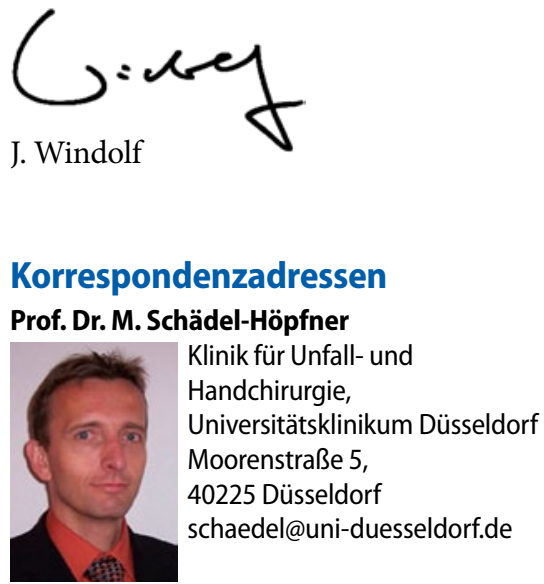

Prof. Dr. Joachim Windolf

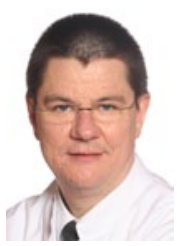

Klinik für Unfall- und Handchirurgie Universitätsklinikum Düsseldorf Moorenstraße 5, 40225 Düsseldorf windolf@uni-duesseldorf.de 